\title{
DISTRIBUTION OF POSITION OF SEGMENT INITIATING THE CHAIN-LIKE STRUCTURE TRANSLOCATION THROUGH THE MEMBRANE OPENING
}

\author{
Kamila Bartlomiejczyk \\ Institute of Mathematics, Czestochowa University of Technology \\ Czestochowa, Poland \\ kamila.bartlomiejczyk@im.pcz.pl
}

\begin{abstract}
This article discusses the transport of chain-like structures across a pore in membrane. Such a translocation process is initiated by the first monomer captured by molecules forming the pore. This study focuses on the distribution of the position of this particular polymer's segment. The polymer attachment process is simulated using the sequential algorithm and the influence of various physical and chemical parameters is analyzed. Selected results are presented by means of different histograms.
\end{abstract}

\section{Introduction}

The translocation of chain-like structures (CLS) through a nanoscopic opening in membranes recently received great attention in experiment, theory and simulations. This interest is caused by the fact that they are widely common in nature. The notion of CLS is encountered in many physical, biological, chemical and technological processes, where it plays a very important role. Among the most interesting examples one can find protein transport through channels in biological membranes, invasion of viruses into cells, gene transfer between bacteria and the motion of DNA and RNA across nuclear pores. Analysis of the latter has wide applications e.g. in the studies of the drug delivery process, gene therapy or rapid DNA sequencing.

Theoretical studies in this field are frequently focused on the fundamental physics of the translocation process as well as on the effects of the pore-polymer interactions, structure of the pore, crowding and confinement effects on the dynamics of translocation. A number of experimental studies have focused on instances where the nanopore captures DNA at one end and then slides it through in a linear, head-to-tail fashion. However, a solid-state nanopore can also capture DNA at some distance from its end and pull it through in a folded configuration. Translocations of folded DNA cause the simultaneous motion of multiple segments across the pore. Therefore it seems interesting to study the molecules at the moment of insertion, which offers clues about how the nanopore captures them. The capture process is important to applications of nanopores that seek to extract sequence- 
-related information from unfolded molecules. Study of polymers at the moment of insertion was already presented in papers, for example in [1].

There are various ways to analyze the motion of polymers and their transport across nanopores in membrane. One of the most common is lattice model, see $[2,3]$. A model like this allows to build a sequential algorithm (for example [3]) which can be easily implemented to computing environment.

\section{The algorithm}

In this paper the problem is analyzed using an algorithm described in [3] that was implemented in Mathematica to create a simulation environment which is based on lattice model. To understand how it works, it is necessary to know some terms and denotations.

Polymers are molecules formed with a large number of covalently bonded small repeating units called monomers. These structures can be seen as long flexible strings because their length is very large compared to the molecular diameter. To describe the polymers and their movements we consider here a two - dimensional (2D) geometry, where a chain position is a finite sequence $c=\left\{c_{1}, c_{2}, \ldots, c_{n}\right\}$ of points $c_{i}=\left(x_{i}, y_{i}\right)$ such that distance $d$ between all consecutive elements is less than given limit $g: d\left(c_{i}, c_{i+1}\right)<g, \quad n=1,2, \ldots, n-1$, where $n$ is a length of polymer. The elements of this collection are called segments' positions in space. They will be identified by their index, i.e. their relative position in the sequence $c$. The segment $\left(c_{n}\right)$ is called a head and $\left(c_{l}\right)$ is called a tail.

The sequential algorithm for chain-like structure movement simulation, see [3], is based on the following assumptions:

Assumption 1. (discretization of motion space): The chain moves along the integer lattice nodes. The movement of the chain (transformation of the chain from one position to another) consists of a sequence of moves.

Assumption 2. (sequentialization of the move): Every single move of a chain can be sequentialized into a sequence of steps (moves made by segments from a node to neighbouring one).

Assumption 3. (chain nature of the move): Every single move of a chain is started by only one segment. Then all segments make their steps sequentially.

Assumption 4. (acceptance of new chain position): The new position of the chain is accepted with a probability on its cost (a function representing fitness connecting with polymer's structure or other properties).

One of the most important notions in the above idea is a selection of the first to move segment (FTMS) that is done according to a given probability distribution. In the simulations presented here it is assumed that the distribution assigns equal probabilities to internal segments, whilst the probabilities of choosing a head and 
of choosing a tail are $a$ times greater. Consequently, the distribution of the FTMS is given by the formula

$$
p_{i}= \begin{cases}\frac{1}{2 a+n-2}, & i=2, \ldots, n-1 \\ \frac{a}{2 a+n-2}, & i=1 \text { and } i=n\end{cases}
$$

The above numbers pi denotes the probability that the i-th segment will be chosen as the FTMS.

\section{Description of the problem and simulation experiments}

The principal aim of the paper is to study the distribution of the relative position of segment initiating the CLS translocation through the membrane opening. This segment will be denoted as SIST.

In our simulations the width of the pore is constant and it equals two nodes. It is assumed that there is potential above the barrier which pulls a segments towards the pore. Consequently the CLS always moves in the direction of the opening. In the simulations an influence of several parameters on the distribution of SIST is examined. Among these parameters are the following: the length of the CLS (n), the distance of the starting CLS position to the barrier (DTB), the FTMS distribution parameter $a$ and the relative position of CLS with respect to the barrier. All these parameters vary in the following ranges. In various previously reported studies the length of polymer varied from a few to 400 segments, see e.g. [2, 4, 5] Therefore, in our simulations, the parameter $n$ is chosen as $n=50,100,500$. A value of parameter $a$ is dependent on the length $n$ and it belongs to the interval $\left[\frac{1}{8} n, n\right]$. The parameter $D T B$ takes the values $D T B=25,50,100,200$ segments. In the simulations two specific relative position of CLS with respect to the barrier are considered: ' $p 1$ ' parallel to the barrier and symmetrically to the hole, ' $p 2$ ' perpendicular to the barrier and in front of the hole. There are 1000 runs made for each setup of specific parameters' values.

\section{Results}

Below we present the probability histograms illustrating the distribution of SIST. In every histogram the horizontal axis presents the relative position of the SIST. As a consequence, the height of bars denotes a probability of touching a pore by a segment with a relative position number given on the x-axis. First we present the results obtained in the simulations performed for CLS that started position is parallel to the barrier and located symmetrically with respect to the opening. 
For $D T B=25$ it could be observed that increasing a value of $n$ and parameter $a$ has an insignificant impact on the SIST distribution. Typical histograms for the distribution of the first segment touching a pore in position ' $p 1$ ' in the case of $D T B=25$ are shown in Figure 1. It can be seen, that the most probable number of SIST in this situation is, approximately, between $\frac{2}{5} n$ and $\frac{3}{5} n$. So, it is generally near the middle of CLS.
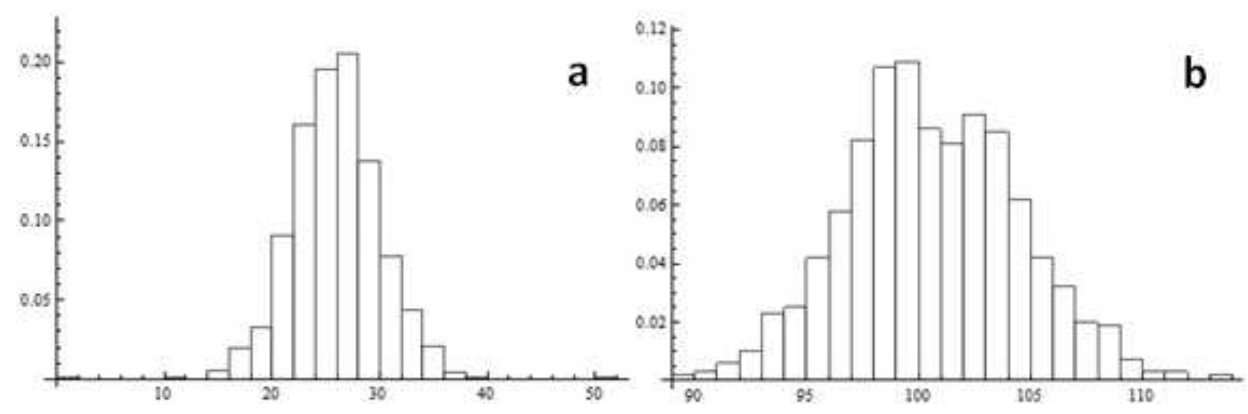

Fig. 1. Distribution of the SIST for (a) $n=50, a=1 / 4 n, D T B=25$, position - 'p1' and (b) $n=200, a=1 / 4 n, D T B=25$, position - 'p1'

It can also be observed that the greater the distance $D T B$, the greater the influence of parameter $a$ on the studied distribution. Overall, the higher $a$, the more frequently the SIST is a tail or a head. It is illustrated in Figure 2a and $2 b$.

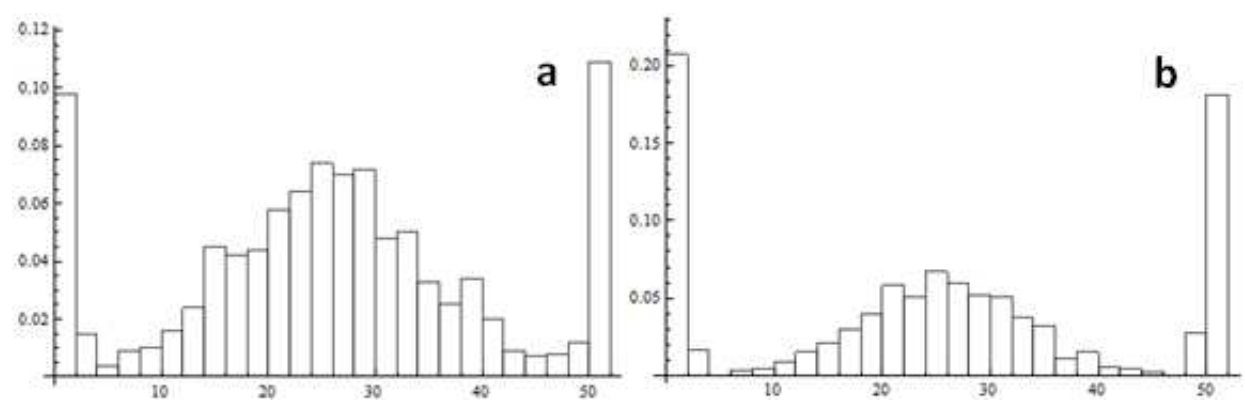

Fig. 2. Distribution of the SIST for (a) $n=50, a=1 / 8 n, D T B=100$, position - 'p1' and (b) $\mathrm{n}=50, \mathrm{a}=\mathrm{n}, \mathrm{DTB}=100$, position - 'p1'

It can also be interesting to analyze an impact of the ratio $r=\frac{D T B}{n}$ on the simulation's results. It can be observed that when $r \geq 1$, a tail or a head of polymer more frequently touches a pore as the first segment (see Fig. 2 in case of $r=2$ ). In other cases the number of the SIST is between $\frac{2}{5} n$ and $\frac{3}{5} n$ (see Fig. 1a in case of $r=\frac{1}{2}$ ).

Based on our results we also compute the basic statistical characteristics of the SIST distribution. It turns out that its mean value is very close to the middle 
segment of the CLS. On the other hand an increase of both, the distance to barrier and the length of CLS results in an increasing standard deviation.

In the next part of our simulations we analyze the situation where the starting position of the CLS is perpendicular with respect to the barrier. As it could be expected in such a case, the most probable number of SIST is $n$ (a head). It can also be noticed that generally the closer to the barrier the starting CLS is, the more often the SIST is a head. An impact of increase of the parameter a is noticeable when $\mathrm{DTB}<2 \mathrm{n}$. For example, let us consider the case DTB $=25$ and $n=50$. The increase of a from $\frac{1}{8} n$ to $\mathrm{n}$ causes growth of fraction of 'a head touching the pore as the first' from 51.6 up to $72.6 \%$ (this situation is shown in Figure 3).
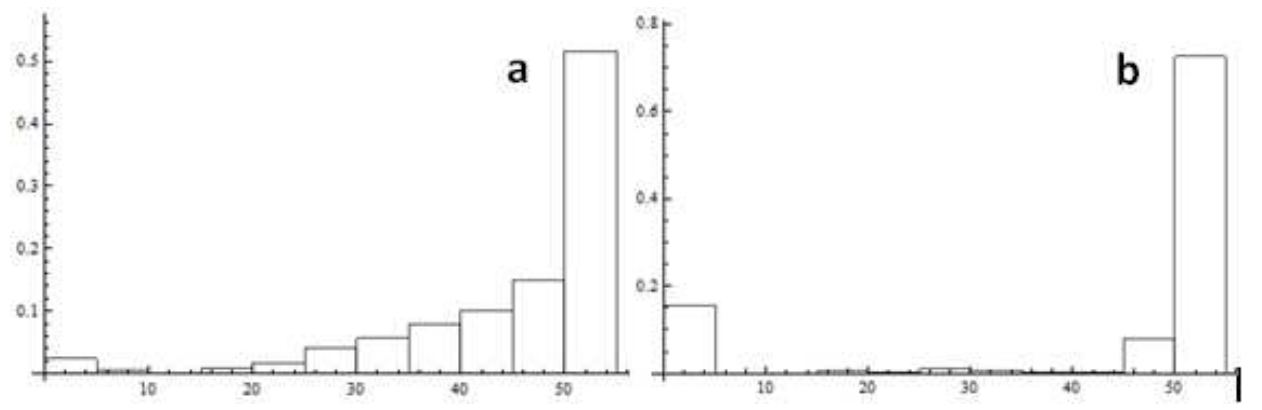

Fig. 3. Distribution of the SIMT for (a) $n=50, a=1 / 8 n, D T B=25$, position - 'p2' and (b) $\mathrm{n}=50, \mathrm{a}=\mathrm{n}, \mathrm{DTB}=25$, position - ' $\mathrm{p} 2$ '

It can also be observed that an increase of the parameter a causes the probability of a tail being the SIST to increase as well, (compare Fig. 3). Moreover, when DTB is increased, a number of cases that a tail inserts a pore as the first segment, is increased too - it is comparable with number of cases that a head is the first. It is shown in Figure 4.

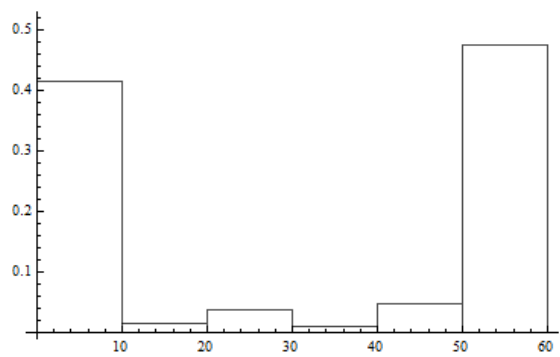

Fig. 4. Distribution of the SIMT for $\mathrm{n}=50, \mathrm{a}=\mathrm{n}, \mathrm{DTB}=200$, position - 'p2'

It should be noticed that if $n>>D T B$, a situation when the SIST is not a tail or a head occurs very rarely. For example, when $D T B=\frac{1}{8} n$ and $\mathrm{a}=\mathrm{n}$, the percentage of cases 'a tail is the first' equals up $83.8 \%$, and of cases 'a head is the first' equals $7.4 \%$. It is worth emphasizing that similar results are obtained for smaller values 
of $a$. Consequently, one can infer that this parameter has an insignificant impact on the SIST distribution when $n>>D T B$.

A mean value of the SIST distribution in case of position 'p2' for small DTB is close to number of a CLS head. An increase of both, the DTB and the parameter a results in decreasing mean value and increasing standard deviation.

\section{Conclusions}

This paper discusses the results of simulations done for selected values of parameters $n, D T B, a$ and for two started positions of polymer - parallel and perpendicular to the barrier. The analysis of the obtained results allows us to draw conclusions about distribution of the relative position of segment initiating the chain-like structure translocation through the membrane opening.

In position ' $\mathrm{p} 1$ ' the ratio $r=\frac{D T B}{n}$ is very important. When $r \geq 1$, the SIST more frequently is a tail or a head (the bigger a, the more frequently). Otherwise the SIST is generally in the range $\left[\frac{2}{5} n, \frac{3}{5} n\right]$. When DTB is too small in comparison with $\mathrm{n}$, the parameter a has an insignificant impact on the distribution of the SIST. So, the higher $r$ the greater an influence of the parameter $a$.

When a polymer initially is in position 'p2' in most cases the SIST is a head (the smaller $D T B$, the more often it happens). An increase of parameter $a$ causes increase of probability of touching a pore by a head (and by a tail also). An important remark is that when $n$ is much greater than $D T B$, inserting a nanopore by another segment than a tail or a head occurs infrequently.

\section{References}

[1] Mihovilovic M., Hagerty N., Stein D., The statistic of DNA capture by a solid-state nanopore, arXiv preprint. 2012, arXiv:1209.3250.

[2] Żurek S., Kośmider M., Drzewiński A., van Leeuwen J.M.J., Translocation of polymers in a lattice model, The European Phys. J. E: Soft Matter and Biological Physics 2012, 35, 47.

[3] Grzybowski A., Domański Z., A sequential algorithm for modelling random movements of chainlike structures, Sci. Res. Inst. Math. 2011, 1(10), 5-10.

[4] Bhattacharya A., Morrison W.H., Luo K., Ala-Nissila A., Ying S., Milchev A., Binder K., Scaling exponents of Forced Polymer Translocation through a nano-pore, The European Phys. J. E: Soft Matt. and Biol. Phys. 2009, 29, 423-429.

[5] Milchev A., Binder K., Bhattacharya A., Polymer translocation through a nanopore induced by adsorption: Monte Carlo simulation of a coarse-grained model, Journal of Chemical Physics $2004,121,12,6042-6051$. 